\title{
Investigating avoidable patient deaths
}

This editorial (BMJ 2017;356:j223, doi:10.1136/bmj.j223) erroneously refers to "Ireland's former chief medical officer, Tony Holohan.” Dr Holohan is actually still in post. 